\title{
A CHARACTERIZATION OF BIFLATNESS OF SEGAL ALGEBRAS BASED ON A CHARACTER
}

\author{
Morteza Essmaili, Mehdi Rostami And Massoud Amini
}

Kharazmi University, Iran; Amirkabir University of Technology, Iran;

Tarbiat Modares University, Iran

Abstract. Let $\mathcal{A}$ be a Banach algebra and $\phi$ be a character on $\mathcal{A}$. In this paper, we give a necessary condition, called condition $(W)$, for $\phi$-biflatness of Banach algebra $\mathcal{A}$ as well as some hereditary properties. We also study the relation between left $\phi$-amenability and condition $(W)$. Moreover, we apply these results and characterize the $\phi$-biflatness of abstract symmetric Segal algebras. In particular, we identify $\phi$-biflatness of the Lebesgue-Fourier algebra $\mathcal{L} A(G)$, where $G$ is a unimodular locally compact group. These results describe a homological property for Segal algebras in the setting of biflatness based on character $\phi$.

\section{INTRODUCTION}

Suppose that $\mathcal{A}$ is a Banach algebra and $E$ is a Banach $\mathcal{A}$-bimodule. We regard the dual space $E^{\prime}$ as a Banach $\mathcal{A}$-bimodule with the operations defined by,

$$
(a \cdot f)(x)=f(x \cdot a), \quad(f \cdot a)(x)=f(a \cdot x) \quad\left(a \in \mathcal{A}, x \in E, f \in E^{\prime}\right) .
$$

We let $\mathcal{A} \hat{\otimes} \mathcal{A}$ denotes the projective tensor product which we view as a Banach $\mathcal{A}$-bimodule with operations determined by:

$$
a \cdot(b \otimes c)=a b \otimes c, \quad(b \otimes c) \cdot a=b \otimes c a \quad(a, b, c \in \mathcal{A}) .
$$

Kaniuth, Lau and Pym have recently introduced and studied in $[10,11]$ the interesting notion of $\phi$-amenability for Banach algebras, where $\phi: \mathcal{A} \longrightarrow$ $\mathbb{C}$ is a character. Simultaneously, M. S. Monfared in [14] introduced and

2010 Mathematics Subject Classification. 16E40, 43A20.

Key words and phrases. $\phi$-biflatness, $\phi$-amenability, group algebras, abstract Segal algebras.

The first and third authors were in part supported by grants from IPM (no. 91470048 \& 91430215). 
investigated the notion of character amenability for Banach algebras. Let $\Delta(\mathcal{A})$ be the set of all characters of the Banach algebra $\mathcal{A}$, and let $\phi \in \Delta(\mathcal{A})$. Following [8], a Banach algebra $\mathcal{A}$ is called left $\phi$-amenable if for all Banach $\mathcal{A}$-bimodules $E$ for which the right module action is given by

$$
x \cdot a=\phi(a) x \quad(a \in \mathcal{A}, x \in E),
$$

every continuous derivation $D: \mathcal{A} \longrightarrow E^{\prime}$ is inner. We say that $\mathcal{A}$ is left character amenable if $\mathcal{A}$ is left $\phi$-amenable for all $\phi \in \Delta(\mathcal{A})$ and has a bounded left approximate identity. Similarly, the right and two-sided version of $\phi$ amenability and character amenability can be defined. These notions have been studied for various classes of Banach algebras. For more details, we refer the reader to $[8,10,11,14]$.

Recently in [17], the authors introduced the notion of $\phi$-biflatness for Banach algebras. A Banach algebra $\mathcal{A}$ is called $\phi$-biflat, if there exists a bounded $\mathcal{A}$-bimodule homomorphism $\rho: \mathcal{A} \longrightarrow(\mathcal{A} \hat{\otimes} \mathcal{A})^{\prime \prime}$ such that

$$
\left(\pi_{\mathcal{A}}^{\prime \prime} \circ \rho(a)\right)(\phi)=\kappa_{\mathcal{A}}(a)(\phi) \quad(a \in \mathcal{A}),
$$

where $\kappa_{\mathcal{A}}: \mathcal{A} \longrightarrow \mathcal{A}^{\prime \prime}$ is the canonical injection on $\mathcal{A}$ and the map $\pi_{\mathcal{A}}$ : $\mathcal{A} \hat{\otimes} \mathcal{A} \longrightarrow \mathcal{A}$ is specified by $\pi_{\mathcal{A}}(a \otimes b)=a b$. They studied some relations between $\phi$-biflatness, $\phi$-amenability and $\phi$-Johnson amenability for Banach algebras. Indeed, It is shown that $\phi$-Johnson amenability of Banach algebras is equivalent to left and right $\phi$-amenability [17, Proposition 2.2]. Moreover, it is proved in [17] that $\phi$-Johnson amenability is equivalent to $\phi$-biflatness and $\phi$-inner amenability. They also characterized $\phi$-biflatness of some classes of Banach algebras associated with locally compact groups. In [18], Samei, Spronk and Stokke studied biflatness of various Segal algebras in both the group algebra, $L^{1}(G)$, and the Fourier algebra, $A(G)$, of a locally compact group $G$. More precisely, they showed that a symmetric Segal algebra $S^{1}(G)$ is flat as a Banach $L^{1}(G)$-bimodule if and only if $G$ is amenable. To our knowledge, there is no characterization of biflatness of symmetric Segal algebras. It is of interest to know whether we obtain anything new if we replace biflatness with $\phi$-biflatness.

The present paper is organized as follows. In section 2, we introduce a new condition on Banach algebras, called condition $(W)$, which is a necessary condition for $\phi$-biflatness of Banach algebras. We obtain some relations between condition $(W)$, left $\phi$-amenability and $\phi$-biflatness. As a consequence, we show that these notions are equivalent for some classes of Banach algebras, such as commutative Banach algebras with a bounded approximate identity. Furthermore, we obtain some hereditary properties of condition $(W)$ and as a main result we show that the semigroup algebra $\ell^{1}\left(\mathbb{N}_{\wedge}\right)$ is character amenable (Theorem 2.10).

In the closing section, section 3 , we examine the condition $(W)$ for some Banach algebras related to the locally compact groups. Indeed, we are able to 
identify $\phi$-biflatness of abstract symmetric Segal algebra $S^{1}(G)$, for a locally compact group $G$, which we believe is interesting in its own right (Theorem 3.4). In the particular case, we conclude that for a unimodular locally compact group $G$, the Lebesgue-Fourier algebra $\mathcal{L} A(G)$, equipped with the convolution product, is $\phi$-biflat if and only if $G$ is amenable.

\section{Condition (W) And $\phi$-Biflatness}

Throughout the paper, $\mathcal{A}$ is a Banach algebra and $\phi \in \Delta(\mathcal{A})$ is a character on $\mathcal{A}$, unless otherwise specified.

In this section, we introduce a new condition on Banach algebra $\mathcal{A}$, which is called condition $(W)$, and study its relation with $\phi$-amenability and $\phi$ biflatness. We show that this property is a necessary condition for $\phi$-biflatness of Banach algebras. Also, we show that for some classes of Banach algebras these notions are equivalent. Moreover, we obtain some hereditary properties related to closed ideals of Banach algebras.

Definition 2.1. We say that $(\mathcal{A}, \phi)$ satisfies condition $(W)$, if there exists a bounded linear operator $\rho: \mathcal{A} \longrightarrow(\mathcal{A} \hat{\otimes} \mathcal{A})^{\prime \prime}$ such that for each $a, b \in \mathcal{A}$,

(1) $\rho(a b)=\phi(a) \rho(b)=\rho(a) \cdot b$,

(2) $\left(\pi_{\mathcal{A}}^{\prime \prime} \circ \rho(a)\right)(\phi)=\phi(a)$.

In the following, we investigate the relation between left $\phi$-amenability and condition $(W)$ on Banach algebras. Moreover, we give an example that shows these notions are not equivalent.

Proposition 2.2. With the above notations,

(i) if $\mathcal{A}$ is left $\phi$-amenable, then $(\mathcal{A}, \phi)$ satisfies condition $(W)$,

(ii) conversely, if $\mathcal{A}$ has a bounded approximate identity and $(\mathcal{A}, \phi)$ satisfies condition $(W)$, then $\mathcal{A}$ is left $\phi$-amenable.

Proof. (i) Since $\mathcal{A}$ is left $\phi$-amenable, by [8, Theorem 2.3] $\mathcal{A}$ has a left $\phi$-virtual diagonal, that is an element $M \in(\mathcal{A} \hat{\otimes} \mathcal{A})^{\prime \prime}$ such that

$$
M \cdot a=\phi(a) M, \quad\left(\pi_{\mathcal{A}}^{\prime \prime}(M)\right)(\phi)=1 \quad(a \in \mathcal{A}) .
$$

Define the map $\rho: \mathcal{A} \longrightarrow(\mathcal{A} \hat{\otimes} \mathcal{A})^{\prime \prime}$ by

$$
\rho(a)=\phi(a) M \quad(a \in \mathcal{A}),
$$

and observe that for each $a, b \in \mathcal{A}$, we have

$$
\rho(a) \cdot b=\phi(a) M \cdot b=\phi(a) \rho(b)=\phi(a) \phi(b) M=\phi(a b) M=\rho(a b) .
$$

On the other hand,

$$
\left(\pi_{\mathcal{A}}^{\prime \prime} \circ \rho(a)\right)(\phi)=\phi(a)\left(\pi_{\mathcal{A}}^{\prime \prime}(M)\right)(\phi)=\phi(a) .
$$

Hence $(\mathcal{A}, \phi)$ satisfies condition $(W)$.

(ii) Suppose that the map $\rho: \mathcal{A} \longrightarrow(\mathcal{A} \hat{\otimes} \mathcal{A})^{\prime \prime}$ is a bounded linear operator satisfying conditions (1) and (2) in Definition 2.1. Let $\left(e_{\alpha}\right)$ be a bounded 
approximate identity for $\mathcal{A}$. By passing to a subnet, we may define $M=$ $w k^{*} \lim \rho\left(e_{\alpha}\right)$. For each $a \in \mathcal{A}$, we have

$$
\begin{aligned}
M \cdot a & =w k^{*} \lim \rho\left(e_{\alpha}\right) \cdot a=w k^{*} \lim \rho\left(e_{\alpha} a\right) \\
& =w k^{*} \lim \rho\left(a e_{\alpha}\right)=\phi(a) w k^{*} \lim \rho\left(e_{\alpha}\right)=\phi(a) M .
\end{aligned}
$$

Moreover,

$$
\pi_{\mathcal{A}}^{\prime \prime}(M)(\phi)=\lim \pi_{\mathcal{A}}^{\prime \prime}\left(\rho\left(e_{\alpha}\right)\right)(\phi)=\lim \phi\left(e_{\alpha}\right)=1 .
$$

Thus $M \in(\mathcal{A} \hat{\otimes} \mathcal{A})^{\prime \prime}$ is a left $\phi$-virtual diagonal for $\mathcal{A}$. By [8, Theorem 2.3], we conclude that $\mathcal{A}$ is left $\phi$-amenable.

REMARK 2.3. We recall that the assumption of the existence of a bounded approximate identity in Proposition 2.2 (ii) can not be removed nor be replaced by the existence of a left identity. For example, let $S$ be a right zero semigroup with $|S| \geq 2$, that is,

$$
s t=t \quad(s, t \in S) .
$$

Let $\phi_{S}: \ell^{1}(S) \longrightarrow \mathbb{C}$ be the augmentation character on the semigroup algebra $\ell^{1}(S)$, defined by

$$
\phi_{S}(f)=\sum_{s \in S} f(s) \quad\left(f \in \ell^{1}(S)\right) .
$$

Fix $t \in S$ and define the map $\rho: \ell^{1}(S) \longrightarrow\left(\ell^{1}(S) \hat{\otimes} \ell^{1}(S)\right)^{\prime \prime}$ by

$$
\rho(f)=\kappa_{\ell^{1}(S)}\left(\delta_{t} \otimes f\right) \quad\left(f \in \ell^{1}(S)\right) .
$$

For each $f, g \in \ell^{1}(S)$, we have

$$
\begin{aligned}
\rho(f * g) & =\rho\left(\phi_{S}(f) g\right)=\phi_{S}(f) \rho(g)=\phi_{S}(f) \delta_{t} \otimes g \\
& =\delta_{t} \otimes(f * g)=\left(\delta_{t} \otimes f\right) \cdot g=\rho(f) \cdot g .
\end{aligned}
$$

Moreover,

$$
\left(\pi^{\prime \prime} \circ \rho(f)\right)\left(\phi_{S}\right)=\kappa_{\ell^{1}(S)}\left(\delta_{t} \otimes f\right)\left(\pi^{\prime}\left(\phi_{S}\right)\right)=\phi_{S}\left(\delta_{t} * f\right)=\phi_{S}(f) .
$$

It follows that $\left(\ell^{1}(S), \phi_{S}\right)$ satisfies condition $(W)$. On the other hand, since the right zero semigroup $S$ is not right amenable it follows from [8, Theorem 2.3 ] that $\ell^{1}(S)$ is not left $\phi_{S}$-amenable.

In the sequel, we prove that the property $(W)$ is a necessary condition for $\phi$-biflatness.

Theorem 2.4. Let $\mathcal{A}$ be a Banach algebra and $\phi \in \Delta(\mathcal{A})$. Consider the following statements:

(i) $\mathcal{A}$ is biflat.

(ii) $\mathcal{A}$ is $\phi$-biflat

(iii) $(\mathcal{A}, \phi)$ satisfies condition $(W)$.

Then we have $(i) \Rightarrow(i i) \Rightarrow(i i i)$. 
Proof. The implication (i) $\Rightarrow$ (ii) is obvious.

(ii) $\Rightarrow$ (iii) Suppose that $\mathcal{A}$ is $\phi$-biflat, so there exists $\rho: \mathcal{A} \longrightarrow(\mathcal{A} \hat{\otimes} \mathcal{A})^{\prime \prime}$ such that for all $a, b \in \mathcal{A}$,

$$
\rho(a b)=a \cdot \rho(b)=\rho(a) \cdot b, \quad\left(\pi_{\mathcal{A}}^{\prime \prime} \circ \rho(a)\right)(\phi)=\phi(a) .
$$

Choose $a_{0} \in \mathcal{A}$ such that $\phi\left(a_{0}\right)=1$. Define the map $T_{\phi, a_{0}}:(\mathcal{A} \hat{\otimes} \mathcal{A})^{\prime} \longrightarrow$ $(\mathcal{A} \hat{\otimes} \mathcal{A})^{\prime}$ by

$$
T_{\phi, a_{0}}(f)=\phi \otimes f\left(a_{0} \otimes \cdot\right) \quad\left(f \in(\mathcal{A} \hat{\otimes} \mathcal{A})^{\prime}\right) .
$$

Now, put $\widetilde{\rho}=T_{\phi, a_{0}}^{\prime} \circ \rho$. Thus, we have

$$
\begin{aligned}
\widetilde{\rho}(a b)(f) & =\rho(a b)\left(T_{\phi, a_{0}}(f)\right)=(a \cdot \rho(b))\left(T_{\phi, a_{0}}(f)\right) \\
& =\rho(b)\left(T_{\phi, a_{0}}(f) \cdot a\right) .
\end{aligned}
$$

On the other hand, for all $c, d \in \mathcal{A}$,

$$
\begin{aligned}
\left(T_{\phi, a_{0}}(f) \cdot a\right)(c \otimes d) & =T_{\phi, a_{0}}(f)(a c \otimes d)=\phi(a c) f\left(a_{0} \otimes d\right) \\
& =\phi(a) \phi(c) f\left(a_{0} \otimes d\right) .
\end{aligned}
$$

By (2.1), we conclude that

$$
\begin{aligned}
\widetilde{\rho}(a b)(f) & =\rho(b)\left(T_{\phi, a_{0}}(f) \cdot a\right)=\rho(b)\left(\phi(a) \phi \otimes f\left(a_{0} \otimes \cdot\right)\right) \\
& =\phi(a) \rho(b)\left(\phi \otimes f\left(a_{0} \otimes \cdot\right)\right)=\phi(a) \rho(b)\left(T_{\phi, a_{0}}(f)\right) \\
& =\phi(a) \widetilde{\rho}(b)(f) .
\end{aligned}
$$

It is easy to see that $T_{\phi, a_{0}}(b \cdot f)=b \cdot T_{\phi, a_{0}}(f)$, thus

$$
\begin{aligned}
(\widetilde{\rho}(a) \cdot b)(f) & =\widetilde{\rho}(a)(b \cdot f)=\rho(a)\left(T_{\phi, a_{0}}(b \cdot f)\right) \\
& =\rho(a)\left(b \cdot T_{\phi, a_{0}}(f)\right)=(\rho(a) \cdot b)\left(T_{\phi, a_{0}}(f)\right) \\
& =\rho(a b)\left(T_{\phi, a_{0}}(f)\right)=\widetilde{\rho}(a b)(f) .
\end{aligned}
$$

Using (2.2) and (2.3), we conclude that $\widetilde{\rho}(a b)=\phi(a) \widetilde{\rho}(b)=\widetilde{\rho}(a) \cdot b$. Moreover,

$$
\begin{aligned}
\widetilde{\phi \circ \pi_{\mathcal{A}}}(c \otimes d) & =\phi(c)\left(\phi \circ \pi_{\mathcal{A}}\left(a_{0} \otimes d\right)\right)=\phi(c) \phi\left(a_{0} d\right) \\
& =\phi(c) \phi(d)=\left(\phi \circ \pi_{\mathcal{A}}\right)(c \otimes d) .
\end{aligned}
$$

Hence, for each $a \in \mathcal{A}$,

$$
\left(\pi_{\mathcal{A}}^{\prime \prime} \circ \widetilde{\rho}(a)\right)(\phi)=\widetilde{\rho}\left(\pi_{\mathcal{A}}^{\prime}(\phi)\right)=\widetilde{\rho}(a)\left(\phi \circ \pi_{\mathcal{A}}\right)=\rho(a)\left(\widetilde{\phi \circ \pi_{\mathcal{A}}}\right) .
$$

Now, using (2.4) and (2.5) we have

$$
\left(\pi_{\mathcal{A}}^{\prime \prime} \circ \widetilde{\rho}(a)\right)(\phi)=\rho(a)\left(\phi \circ \pi_{\mathcal{A}}\right)=\left(\pi_{\mathcal{A}}^{\prime \prime} \circ \rho(a)\right)(\phi)=\phi(a) .
$$

It follows that $(\mathcal{A}, \phi)$ satisfies condition $(W)$.

REMARK 2.5. We note that in the above theorem the implication (iii) $\Rightarrow$ (i) does not hold in general. For example, any $C^{*}$-algebra $\mathcal{A}$ is left $\phi$-amenable for each $\phi \in \Delta(\mathcal{A})$ [10, Proposition 2.1] and so it satisfies condition $(W)$, by Proposition 2.2. However, it is well known that $\mathcal{A}$ is not biflat, if it is not nuclear. A concrete example is the algebra of bounded operators on any 
infinite dimensional Hilbert space. As another example, It showed in [9] that $A(S O(3))$ is unital non-amenable and so is not biflat. However, $A(S O(3))$ is $\phi$-amenable, for each $\phi \in \Delta(A(S O(3)))$, see [10, Example 2.6].

In [17], the authors introduced the notion of $\phi$-Johnson amenability of Banach algebras. A Banach algebra $\mathcal{A}$ is $\phi$-Johnson amenable if there exists an element $\mathbf{m} \in(\mathcal{A} \hat{\otimes} \mathcal{A})^{\prime \prime}$ such that $a \cdot \mathbf{m}=\mathbf{m} \cdot a$ and $\left(\pi_{\mathcal{A}}^{\prime \prime}(\mathbf{m})\right)(\phi)=1$, for each $a \in \mathcal{A}$. It is shown in [17, Proposition 2.2] that $\phi$-Johnson amenability is equivalent to left and right $\phi$-amenability. They also proved that $\phi$-Johnson amenability is a sufficient condition for $\phi$-biflatness [17, Lemma 3.1]. Therefore, we have the following implications:

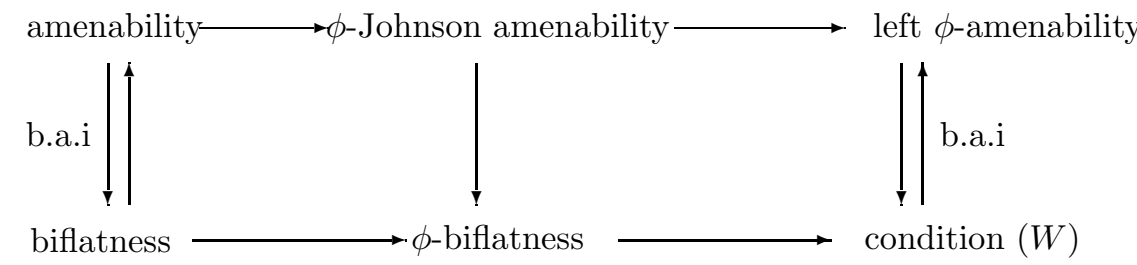

As a consequence of Theorem 2.4 and Proposition 2.2, we conclude that $\phi$ amenability, $\phi$-biflatness and condition $(W)$ are equivalent for a certain class of Banach algebras.

Corollary 2.6. Let $\mathcal{A}$ be a commutative Banach algebra with a bounded approximate identity and $\phi \in \Delta(\mathcal{A})$. Then the following are equivalent:

(i) $\mathcal{A}$ is $\phi$-biflat.

(ii) $(\mathcal{A}, \phi)$ satisfies condition $(W)$.

(iii) $\mathcal{A}$ is $\phi$-amenable.

Proof. The implications (i) $\Rightarrow$ (ii) and (ii) $\Rightarrow$ (iii) follow from Theorem 2.4 and Proposition 2.2 (ii), respectively.

For (iii) $\Rightarrow(\mathrm{i})$, we note that $\phi$-amenability of $\mathcal{A}$ is equivalent to $\phi$-Johnson amenability of $\mathcal{A}[17$, Proposition 2.2$]$. Now, by [17, Lemma 3.1] we conclude that $\mathcal{A}$ is $\phi$-biflat and the proof is complete.

EXAMPLE 2.7. Let $\ell^{1}\left(\mathbb{N}_{\vee}\right)$ be the semigroup algebra on semigroup $S=$ $(\mathbb{N}, \vee)$ with the following product:

$$
\mathbb{N} \times \mathbb{N} \longrightarrow \mathbb{N}, \quad(m, n) \longrightarrow m \vee n=\max \{m, n\} .
$$

In [17, Example 5.5], it is shown that the semigroup algebra $\ell^{1}\left(\mathbb{N}_{\vee}\right)$ is $\phi$-biflat for each $\phi \in \Delta\left(\ell^{1}\left(\mathbb{N}_{\vee}\right)\right)$. As an immediate consequence of Corollary 2.6, since the semigroup algebra $\ell^{1}\left(\mathbb{N}_{\vee}\right)$ is commutative, unital and by [2, Corollary 2.2] is character amenable, we obtain the similar result.

In the sequel, we study some hereditary properties of condition $(W)$ related to closed ideals. For this aim, we need the following Lemma. For $\phi \in \Delta(\mathcal{A})$, 
we recall that the map $\phi \otimes \phi: \mathcal{A} \hat{\otimes} \mathcal{A} \longrightarrow \mathbb{C}$ is defined by,

$$
(\phi \otimes \phi)(a \otimes b)=\phi(a) \phi(b) \quad(a, b \in \mathcal{A}) .
$$

Lemma 2.8. Let $\mathcal{A}$ be a Banach algebra. The following conditions are equivalent:

(i) $(\mathcal{A}, \phi)$ satisfies condition $(W)$.

(ii) There exists a bounded linear map $\sigma:(\mathcal{A} \hat{\otimes} \mathcal{A})^{\prime} \longrightarrow \mathcal{A}^{\prime}$ such that for each $a \in \mathcal{A}$ and $f \in(\mathcal{A} \hat{\otimes} \mathcal{A})^{\prime}$,

$$
\sigma(a \cdot f)=a \cdot \sigma(f), \quad \phi(a) \sigma(f)=\sigma(f) \cdot a
$$

and $\left(\pi_{\mathcal{A}}^{\prime} \circ \sigma\right)(\phi \otimes \phi)=\phi \otimes \phi$.

Proof. (i) $\Rightarrow$ (ii) By assumption, there is a bounded linear map $\rho: \mathcal{A} \longrightarrow$ $(\mathcal{A} \hat{\otimes} \mathcal{A})^{\prime \prime}$ such that

$$
\rho(a b)=\phi(a) \rho(b)=\rho(a) \cdot b, \quad\left(\pi_{\mathcal{A}}^{\prime \prime} \circ \rho(a)\right)(\phi)=\phi(a),
$$

for all $a, b \in \mathcal{A}$. Define the map $\sigma:(\mathcal{A} \hat{\otimes} \mathcal{A})^{\prime} \longrightarrow \mathcal{A}^{\prime}$ by $\sigma=\rho_{\left.\right|_{(\mathcal{A} \hat{\otimes} \mathcal{A})^{\prime}} ^{\prime}}$. It is easy to see that $\sigma$ satisfies (ii).

For (ii) $\Rightarrow$ (i), it suffices to put $\rho=\sigma_{\left.\right|_{\mathcal{A}} ^{\prime}}^{\prime}$.

THEOREM 2.9. Let I be a closed right ideal of $\mathcal{A}$ and $\phi \in \Delta(\mathcal{A})$ such that $\phi_{\left.\right|_{I}} \neq 0$.

(i) If $(\mathcal{A}, \phi)$ satisfies condition $(W)$, then so does $\left(I, \phi_{\left.\right|_{I}}\right)$.

(ii) Conversely, if $I$ has a right approximate identity and $\left(I, \phi_{\mid I}\right)$ satisfies condition $(W)$, then so is $(\mathcal{A}, \phi)$.

Proof. (i) Let $\rho:(\mathcal{A} \hat{\otimes} \mathcal{A})^{\prime} \longrightarrow \mathcal{A}^{\prime}$ be the bounded linear map satisfying in Lemma 2.8. Take $b_{0} \in I$ such that $\phi\left(b_{0}\right)=1$ and define the map $\sigma$ : $(I \hat{\otimes} I)^{\prime} \longrightarrow I^{\prime}$ by

$$
\sigma(f)(b)=\rho(\widetilde{f})(b) \quad\left(f \in(I \hat{\otimes} I)^{\prime}, b \in I\right),
$$

where $\tilde{f}: \mathcal{A} \hat{\otimes} \mathcal{A} \longrightarrow \mathbb{C}$ is defined by

$$
\widetilde{f}\left(a_{1} \otimes a_{2}\right)=f\left(b_{0} a_{1} \otimes b_{0} a_{2}\right) \quad\left(a_{1}, a_{2} \in \mathcal{A}\right) .
$$

For each $a, b \in I$ we have,

$$
\sigma(a \cdot f)(b)=\rho(\widetilde{a \cdot f})(b)=\rho(a \cdot \widetilde{f})(b)=(a \cdot \rho(\widetilde{f}))(b),
$$

and

$(\sigma(f) \cdot a)(b)=\sigma(f)(a b)=\rho(\widetilde{f})(a b)=\rho(\tilde{f} \cdot a)(b)=\phi(a) \rho(\widetilde{f})(b)=\phi(a) \sigma(f)(b)$.

Moreover,

$$
\begin{aligned}
\left(\pi_{I}^{\prime} \circ \sigma\left(\phi_{I} \otimes \phi_{I}\right)\right)(a \otimes b) & =\sigma\left(\phi_{I} \otimes \phi_{I}\right)(a b)=\rho\left(\widetilde{\phi_{I} \otimes \phi_{I}}\right)(a b)=\rho(\phi \otimes \phi)(a b) \\
& =\left(\pi_{\mathcal{A}}^{\prime} \circ \rho(\phi \otimes \phi)\right)(a \otimes b)=(\phi \otimes \phi)(a \otimes b) .
\end{aligned}
$$


So, we conclude that $\sigma$ satisfies conditions of Lemma 2.8 and the proof is complete.

(ii) Since $\left(I, \phi_{\left.\right|_{I}}\right)$ satisfies condition $(W)$, by Lemma 2.8 there exists a bounded linear map $\sigma:(I \hat{\otimes} I)^{\prime} \longrightarrow I^{\prime}$ such that

$$
\sigma(b \cdot f)=b \cdot \sigma(f), \quad \sigma(f) \cdot b=\phi(b) \sigma(f) \quad\left(f \in(I \hat{\otimes} I)^{\prime}, b \in I\right),
$$

and $\pi_{I}^{\prime} \circ \sigma\left(\phi_{I} \otimes \phi_{I}\right)=\phi_{I} \otimes \phi_{I}$. Take $b_{0} \in I$ such that $\phi\left(b_{0}\right)=1$ and define $\rho:(\mathcal{A} \hat{\otimes} \mathcal{A})^{\prime} \longrightarrow \mathcal{A}^{\prime}$ by

$$
\rho(f)(a)=\sigma(\tilde{f})\left(b_{0} a\right) \quad\left(f \in(\mathcal{A} \hat{\otimes} \mathcal{A})^{\prime}, a \in \mathcal{A}\right),
$$

where $\tilde{f}=f_{\left.\right|_{I \hat{\otimes} I}}$. Let $\left(e_{\alpha}\right)$ be a right approximate identity for $I$, then for each $a_{1}, a_{2} \in \mathcal{A}$ we have,

$$
\begin{gathered}
\rho\left(a_{1} \cdot f\right)\left(a_{2}\right)=\sigma\left(\widetilde{a_{1} \cdot f}\right)\left(b_{0} a_{2}\right)=\lim _{\alpha} \sigma\left(\widetilde{a_{1} \cdot f}\right)\left(b_{0} a_{2} e_{\alpha}\right) \\
(2.6) \quad=\lim _{\alpha} \sigma\left(e_{\alpha} a_{1} \cdot \widetilde{f}\right)\left(b_{0} a_{2}\right)=\lim _{\alpha}\left(e_{\alpha} a_{1} \cdot \sigma(\widetilde{f})\right)\left(b_{0} a_{2}\right) \\
\quad=\lim _{\alpha} \sigma(\widetilde{f})\left(b_{0} a_{2} e_{\alpha} a_{1}\right)=\sigma(\widetilde{f})\left(b_{0} a_{2} a_{1}\right)=\rho(f)\left(a_{2} a_{1}\right)=a_{1} \cdot \rho(f)\left(a_{2}\right),
\end{gathered}
$$

and

$$
\begin{aligned}
& \left(\rho(f) \cdot a_{1}\right)\left(a_{2}\right)=\sigma(\widetilde{f})\left(b_{0} a_{1} a_{2}\right)=\lim _{\alpha} \sigma(\widetilde{f})\left(b_{0} a_{1} e_{\alpha} a_{2}\right) \\
& \quad=\lim _{\alpha} \phi\left(a_{1}\right) \sigma(\widetilde{f})\left(e_{\alpha} a_{2}\right)=\lim _{\alpha} \phi\left(a_{1}\right)\left(\sigma(\widetilde{f}) \cdot b_{0}\right)\left(e_{\alpha} a_{2}\right) \\
& \quad=\phi\left(a_{1}\right) \lim \sigma(\widetilde{f})\left(b_{0} e_{\alpha} a_{2}\right)=\phi\left(a_{1}\right) \sigma(\widetilde{f})\left(b_{0} a_{2}\right)=\phi\left(a_{1}\right) \rho(f)\left(a_{2}\right) .
\end{aligned}
$$

Moreover,

$$
\begin{aligned}
\left(\pi_{\mathcal{A}}^{\prime} \circ \rho(\phi\right. & \otimes \phi))\left(a_{1} \otimes a_{2}\right)=\rho(\phi \otimes \phi)\left(a_{1} a_{2}\right)=\sigma(\widetilde{\phi \otimes \phi})\left(b_{0} a_{1} a_{2}\right) \\
& =\lim _{\alpha} \sigma(\widetilde{\phi \otimes \phi})\left(b_{0} a_{1} e_{\alpha} a_{2}\right)=\lim _{\alpha} \pi_{\mathcal{A}} \circ \sigma(\widetilde{\phi \otimes \phi})\left(b_{0} a_{1} \otimes e_{\alpha} a_{2}\right) \\
& =\lim _{\alpha}\left(\phi_{\left.\right|_{I}} \otimes \phi_{\left.\right|_{I}}\right)\left(b_{0} a_{1} \otimes e_{\alpha} a_{2}\right)=\lim _{\alpha} \phi\left(b_{0} a_{1}\right) \phi\left(e_{\alpha} a_{2}\right) \\
& =\lim _{\alpha} \phi\left(b_{0} a_{1} e_{\alpha} a_{2}\right)=\phi\left(b_{0} a_{1} a_{2}\right)=(\phi \otimes \phi)\left(a_{1} \otimes a_{2}\right) .
\end{aligned}
$$

Now, using the equations $(2.6),(2.7),(2.8)$ and Lemma 2.8, we conclude that $(\mathcal{A}, \phi)$ satisfies condition $(W)$.

In the following theorem, we show that the semigroup algebra $\ell^{1}\left(\mathbb{N}_{\wedge}\right)$ is character amenable and satisfies condition $(W)$.

ThEOREM 2.10. Suppose that $\ell^{1}\left(\mathbb{N}_{\wedge}\right)$ is the semigroup algebra on semigroup $S=(\mathbb{N}, \wedge)$ with the product defined by $m \wedge n=\min \{m, n\}$. Then, we have all following assertions:

(i) $\left(\ell^{1}\left(\mathbb{N}_{\wedge}\right), \phi\right)$ satisfies condition $(W)$, for each $\phi \in \Delta\left(\ell^{1}\left(\mathbb{N}_{\wedge}\right)\right)$.

(ii) $\ell^{1}\left(\mathbb{N}_{\wedge}\right)$ is character amenable.

(iii) $\ell^{1}\left(\mathbb{N}_{\wedge}\right)$ is $\phi$-biflat, for each $\phi \in \Delta\left(\ell^{1}\left(\mathbb{N}_{\wedge}\right)\right)$. 
Proof. Since $\ell^{1}\left(\mathbb{N}_{\wedge}\right)$ is a commutative Banach algebra with the bounded approximate identity $\left(\delta_{n}\right)_{n \in \mathbb{N}}$, by Corollary 2.6 it suffices to show that the statement (i) holds. It is easy to verify that $\Delta\left(\ell^{1}\left(\mathbb{N}_{\wedge}\right)\right)=\left\{\phi_{n}: n \in \mathbb{N}\right\}$, where for each $n \in \mathbb{N}$ the map $\phi_{n}: \ell^{1}\left(\mathbb{N}_{\wedge}\right) \longrightarrow \mathbb{C}$ is defined by

$$
\phi_{n}(f)=\sum_{i=n}^{\infty} f(i), \quad\left(f \in \ell^{1}\left(\mathbb{N}_{\wedge}\right)\right) .
$$

For each $n \in \mathbb{N}$, we also suppose that $I_{n}$ is the ideal of $\ell^{1}\left(\mathbb{N}_{\wedge}\right)$ generated by the set $\left\{\delta_{1}, \delta_{2}, \delta_{3}, \ldots, \delta_{n}\right\}$. In [17, Example 5.2], it is shown that $\ell^{1}\left(\mathbb{N}_{\wedge}\right)$ is $\phi_{1}$-biflat and so $\left(\ell^{1}\left(\mathbb{N}_{\wedge}\right), \phi_{1}\right)$ satisfies condition $(W)$.

In the case where $n \geq 2$, it follows from [2, Theorem 2.1] that $I_{n}$ is $\phi_{\left.\right|_{I_{n}}}$ amenable and so $\left(I_{n}, \phi_{\left.\right|_{I_{n}}}\right)$ satisfies condition $(W)$. Thus by Theorem 2.9 (ii), we conclude that $\left(\ell^{1}\left(\mathbb{N}_{\wedge}\right), \phi_{n}\right)$ satisfies condition $(W)$ and the proof is complete.

\section{SOME RESUlts In HARMONIC ANALYSIS}

Let $G$ be a locally compact group with a fixed left Haar measure $\lambda_{G}$ and consider the group algebra $L^{1}(G)$ and the measure algebra $M(G)$. It well known that $L^{1}(G)$ is a two-sided ideal with a bounded approximate identity in $M(G)$; see [7] for more details. Let $\widehat{G}$ be the set of all continuous homomorphism from $G$ into the circle group $\mathbb{T}$. For $\rho \in \widehat{G}$, define $\phi_{\rho}$ to be the character induced by $\rho$ on $L^{1}(G)$,

$$
\phi_{\rho}(f)=\int f(x) \overline{\rho(x)} d \lambda_{G}(x) \quad\left(f \in L^{1}(G)\right) .
$$

It is well-known that

$$
\Delta\left(L^{1}(G)\right)=\left\{\phi_{\rho}: \rho \in \widehat{G}\right\},
$$

see for example [7, Theorem 23.7]. We denote the unique extension of $\phi_{\rho}$ to a character on $M(G)$ with the same notation. As a consequence of Theorem 2.9 , we have the following result.

Corollary 3.1. Let $G$ be a locally compact group and $\phi_{\rho} \in \Delta\left(L^{1}(G)\right)$. Then the following statements are equivalent:

(i) $L^{1}(G)$ is $\phi_{\rho}$-biflat.

(ii) $\left(L^{1}(G), \phi_{\rho}\right)$ satisfies condition $(W)$.

(iii) $\left(M(G), \phi_{\rho}\right)$ satisfies condition $(W)$.

(iv) $G$ is an amenable group.

Proof. The implications (i) $\Rightarrow$ (ii) and (iv) $\Rightarrow$ (i) are trivial. The equivalence of (ii) and (iii) follows from Theorem 2.9. Moreover, the implication (ii) $\Rightarrow$ (iv) follows by applying Proposition 2.2 (ii) and [1, Corollary 3.4]. 
In the sequel, we consider condition $(W)$ for abstract Segal algebras. We also apply these results to Segal algebras and Lebesgue-Fourier algebras of a locally compact group $G$.

Definition 3.2. Suppose that $\left(\mathcal{B},\|\cdot\|_{\mathcal{B}}\right)$ and $\left(\mathcal{A},\|\cdot\|_{\mathcal{A}}\right)$ are two Banach algebras such that $\mathcal{B} \subseteq \mathcal{A}$ and

(i) $\mathcal{B}$ is a two-sided dense ideal,

(ii) there exists $M>0$ such that for each $a \in \mathcal{B},\|a\|_{\mathcal{A}} \leq M\|a\|_{\mathcal{B}}$,

(iii) there exists $C>0$ such that for each $a \in \mathcal{A}$ and $b \in \mathcal{B}$,

$$
\|a b\|_{\mathcal{B}},\|b a\|_{\mathcal{B}} \leq C\|a\|_{\mathcal{A}}\|b\|_{\mathcal{B}}
$$

Then we say that $\mathcal{B}$ is a symmetric abstract Segal algebra in $\mathcal{A}$.

Suppose that $\mathcal{B}$ is a symmetric abstract Segal algebra in $\mathcal{A}$. By $[1$, Lemma $2.2]$

$$
\Delta(\mathcal{B})=\left\{\phi_{\left.\right|_{\mathcal{B}}}: \phi \in \Delta(\mathcal{A})\right\} .
$$

TheOREM 3.3. Let $\mathcal{B}$ be a symmetric abstract Segal algebra in $\mathcal{A}$ and $\phi \in \Delta(\mathcal{A})$. Then the following are equivalent:

(i) $\left(\mathcal{B}, \phi_{\left.\right|_{\mathcal{B}}}\right)$ satisfies condition $(W)$.

(ii) $(\mathcal{A}, \phi)$ satisfies condition $(W)$.

Proof. (i) $\Rightarrow$ (ii). By Lemma 2.8, there exists a bounded linear map $\sigma:(\mathcal{B} \hat{\otimes} \mathcal{B})^{\prime} \longrightarrow \mathcal{B}^{\prime}$ such that for each $b \in \mathcal{B}$,

$$
\sigma(b \cdot f)=b \cdot \sigma(f), \quad \phi(b) \sigma(f)=\sigma(f) \cdot b,
$$

and $\left(\pi_{\mathcal{B}}^{\prime} \circ \sigma\right)\left(\phi_{\left.\right|_{\mathcal{B}}} \otimes \phi_{\left.\right|_{\mathcal{B}}}\right)=\phi_{\left.\right|_{\mathcal{B}}} \otimes \phi_{\left.\right|_{\mathcal{B}}}$. For $f \in(\mathcal{A} \hat{\otimes} \mathcal{A})^{\prime}$ put $\tilde{f}=f_{\left.\right|_{(\mathcal{B} \otimes \hat{B})}}$. For each $b_{1}, b_{2} \in \mathcal{B}$,

$$
\begin{aligned}
\left|\widetilde{f}\left(b_{1} \otimes b_{2}\right)\right| & =\left|f\left(b_{1} \otimes b_{2}\right)\right| \leq\|f\|\left\|b_{1} \otimes b_{2}\right\|_{\pi_{\mathcal{A}}} \\
& \leq\|f\|\left\|b_{1}\right\|_{\mathcal{B}}\left\|b_{2}\right\|_{\mathcal{B}} M^{2}=M^{2}\|f\|\left\|b_{1} \otimes b_{2}\right\|_{\pi_{\mathcal{B}}} .
\end{aligned}
$$

Take $b_{0} \in \mathcal{B}$ such that $\phi\left(b_{0}\right)=1$ and define $\rho:(\mathcal{A} \hat{\otimes} \mathcal{A})^{\prime} \longrightarrow \mathcal{A}^{\prime}$ by

$$
\rho(f)(a)=\sigma(\widetilde{f})\left(b_{0} a\right) \quad\left(f \in(\mathcal{A} \hat{\otimes} \mathcal{A})^{\prime}, a \in \mathcal{A}\right) .
$$

For each $a_{1}, a_{2} \in \mathcal{A}$,

$$
\begin{aligned}
& \left(\pi^{\prime} \circ \rho(\phi \otimes \phi)\right)\left(a_{1} \otimes a_{2}\right)=\rho(\phi \otimes \phi)\left(a_{1} a_{2}\right)=\sigma(\widetilde{\phi \otimes \phi})\left(b_{0} a_{1} a_{2}\right) \\
& \quad=\sigma\left(\phi_{\left.\right|_{\mathcal{B}}} \otimes \phi_{\left.\right|_{\mathcal{B}}}\right)\left(b_{0} a_{1} a_{2}\right)=\sigma\left(b_{0} \cdot\left(\phi_{\left.\right|_{\mathcal{B}}} \otimes \phi_{\left.\right|_{\mathcal{B}}}\right)\right)\left(b_{0} a_{1} a_{2}\right) \\
& \quad=\left(b_{0} \cdot \sigma\left(\phi_{\left.\right|_{\mathcal{B}}} \otimes \phi_{\left.\right|_{\mathcal{B}}}\right)\right)\left(b_{0} a_{1} a_{2}\right)=\sigma\left(\phi_{\left.\right|_{\mathcal{B}}} \otimes \phi_{\left.\right|_{\mathcal{B}}}\right)\left(b_{0} a_{1} a_{2} b_{0}\right) \\
& \quad=\left(\phi_{\left.\right|_{\mathcal{B}}} \otimes \phi_{\left.\right|_{\mathcal{B}}}\right)\left(b_{0} a_{1} \otimes a_{2} b_{0}\right)=\phi\left(a_{1}\right) \phi\left(a_{2}\right)=(\phi \otimes \phi)\left(a_{1} \otimes a_{2}\right) .
\end{aligned}
$$


On the other hand, Since $\mathcal{B}$ is a dense ideal of $\mathcal{A}$, there is a sequence $\left(b_{n}\right)$ in $\mathcal{B}$ such that $b_{n} \longrightarrow a_{2}$, thus

$$
\begin{aligned}
& \left(\rho\left(f \partial_{1}\right)\left(a_{2}\right)=\rho(f)\left(a_{1} a_{2}\right)=\sigma(\widetilde{f})\left(b_{0} a_{1} a_{2}\right)=\lim _{n} \sigma(\widetilde{f})\left(b_{0} a_{1} b_{n}\right)\right. \\
& \quad=\lim _{n}\left(\sigma(\widetilde{f}) \cdot b_{0} a_{1}\right)\left(b_{n}\right)=\phi\left(b_{0} a_{1}\right) \lim _{n} \sigma(\widetilde{f})\left(b_{n}\right) \\
& =\phi\left(a_{1}\right) \lim _{n}\left(\sigma(\widetilde{f}) \cdot b_{0}\right)\left(b_{n}\right)=\phi\left(a_{1}\right) \sigma(\widetilde{f})\left(b_{0} a_{2}\right)=\phi\left(a_{1}\right) \sigma(\widetilde{f})\left(a_{2}\right) .
\end{aligned}
$$

Moreover, $b_{n} \cdot f \longrightarrow a \cdot f$ and so

$$
\begin{aligned}
\rho\left(a_{1} \cdot f\right)\left(a_{2}\right) & =\sigma(\widetilde{a \cdot f})\left(b_{0} a_{2}\right)=\sigma(a \cdot \widetilde{f})\left(b_{0} a_{2}\right)=\lim _{n} \sigma\left(b_{n} \cdot \widetilde{f}\right)\left(b_{0} a_{2}\right) \\
& =\lim _{n}\left(b_{n} \cdot \sigma(\widetilde{f})\right)\left(b_{0} a_{2}\right)=\lim _{n} \sigma(\widetilde{f})\left(b_{0} a_{2} b_{n}\right)=\sigma(\widetilde{f})\left(b_{0} a_{2} a_{1}\right) \\
& =\rho(f)\left(a_{2} a_{1}\right)=\left(a_{1} \cdot \rho(f)\right)\left(a_{2}\right) .
\end{aligned}
$$

By equations (3.1), (3.2) and (3.3), we conclude that $(\mathcal{A}, \phi)$ satisfies condition $(W)$.

(ii) $\Rightarrow$ (i) By Lemma 2.8, there exists a bounded linear map $\sigma:(\mathcal{A} \hat{\otimes} \mathcal{A})^{\prime} \longrightarrow$ $\mathcal{A}^{\prime}$ such that for each $a \in \mathcal{A}$,

$$
\sigma(a \cdot f)=a \cdot \sigma(f), \quad \phi(a) \sigma(f)=\sigma(f) \cdot a,
$$

and $\left(\pi_{\mathcal{A}}^{\prime} \circ \sigma\right)(\phi \otimes \phi)=\phi \otimes \phi$. For $f \in(\mathcal{B} \otimes \hat{B})^{\prime}$ define $\widetilde{f} \in(\mathcal{A} \hat{\otimes} \mathcal{A})^{\prime}$ by $\widetilde{f}\left(a_{1} \otimes a_{2}\right)=$ $f\left(a_{1} b_{0} \otimes b_{0} a_{2}\right)$, where $b_{0} \in \mathcal{B}$ such that $\phi\left(b_{0}\right)=1$. For each $a_{1}, a_{2} \in \mathcal{A}$,

$$
\begin{aligned}
\left|\widetilde{f}\left(a_{1} \otimes a_{2}\right)\right| & =\left|f\left(a_{1} b_{0} \otimes b_{0} a_{2}\right)\right| \leq\|f\|\left\|a_{1} b_{0} \otimes b_{0} a_{2}\right\|_{\pi_{\mathcal{B}}} \\
& \leq\|f\|\left\|a_{1} b_{0}\right\|_{\mathcal{B}}\left\|a_{2} b_{0}\right\|_{\mathcal{B}} C^{2} \leq C^{2}\|f\|\left\|b_{0}\right\|^{2}\left\|a_{1} \otimes a_{2}\right\|_{\pi_{\mathcal{A}}} .
\end{aligned}
$$

Now define $\rho:(\mathcal{B} \hat{\otimes} \mathcal{B})^{\prime} \longrightarrow \mathcal{B}^{\prime}$ by

$$
\rho(f)(b)=\sigma(\widetilde{f})(b) \quad\left(f \in(\mathcal{B} \hat{\otimes} \mathcal{B})^{\prime}, b \in \mathcal{B}\right) .
$$

For each $b_{1}, b_{2} \in \mathcal{B}$,

$$
\begin{aligned}
& \left.\pi^{\prime} \circ \rho\left(\left.\left.\phi\right|_{\mathcal{B} \otimes \phi}\right|_{\mathcal{B}}\right)\right)\left(b_{1} \otimes b_{2}\right)=\rho(\phi \otimes \phi)\left(b_{1} b_{2}\right) \\
& \quad=\sigma(\widetilde{\phi \otimes \phi})\left(b_{1} b_{2}=\pi_{\mathcal{A}}^{\prime} \circ \sigma(\phi \otimes \phi)\left(b_{1} \otimes b_{2}\right)=(\phi \otimes \phi)\left(b_{1} \otimes b_{2}\right) .\right.
\end{aligned}
$$

Moreover, for each $b_{1}, b_{2} \in \mathcal{B}$ and $f \in(\mathcal{B} \hat{\otimes} \mathcal{B})^{\prime}$ we have

$$
\begin{aligned}
\rho\left(b_{1} \cdot f\right)\left(b_{2}\right) & =\sigma\left(\widetilde{b_{1} \cdot f}\right)\left(b_{2}\right)=\sigma\left(b_{1} \cdot \widetilde{f}\right)\left(b_{2}\right) \\
& =b_{1} \cdot \sigma(\widetilde{f})\left(b_{2}\right)=\left(b_{1} \cdot \rho(f)\right)\left(b_{2}\right),
\end{aligned}
$$

and

$$
\begin{aligned}
\left(\rho(f) \cdot b_{1}\right)\left(b_{2}\right) & =\rho(f)\left(b_{1} b_{2}\right)=\sigma(\widetilde{f})\left(b_{1} b_{2}\right) \\
& =\sigma(\widetilde{f}) \cdot b_{1}\left(b_{2}\right)=\phi\left(b_{1}\right) \rho(f)\left(b_{2}\right) .
\end{aligned}
$$

By equations (3.4), (3.5) and (3.6), we conclude that $\left(\mathcal{B},\left.\phi\right|_{\mathcal{B}}\right)$ satisfies condition $(W)$. 
Recently, E. Samei, N. Spronk and R. Stokke in [18] gave a detailed study of some homological properties of modules over the group algebra of a locally compact group $G$. In their work they focused on flatness of the Segal algebra $S^{1}(G)$ as a $L^{1}(G)$-bimodule. They were able to show, for example, that when viewed as a $L^{1}(G)$-bimodule, the symmetric Segal algebra $S^{1}(G)$ is flat precisely when the group $G$ is amenable. In the following, we characterize $\phi$ biflatness of symmetric Segal algebras on a locally compact group. But first, we need to recall the following terminologies:

Let $G$ be a locally compact group. A Banach space $\left(S^{1}(G),\|\cdot\|_{S}\right)$ is a Segal algebra on $G$ if it satisfies the following conditions:

(i) $S^{1}(G)$ is dense in $L^{1}(G)$,

(ii) there exist $M>0$ such that $\|f\|_{1} \leq M\|f\|_{S}$ for all $f \in S^{1}(G)$,

(iii) $S^{1}(G)$ is left translation invariant and the map $x \mapsto \delta_{x} * f, G \rightarrow S^{1}(G)$, is continuous for all $f \in S^{1}(G)$,

(iv) $\left\|\delta_{x} * f\right\|_{S}=\|f\|_{S}$, for all $f \in S^{1}(G)$ and $x \in G$.

A Segal algebra is symmetric if it is right translation invariant and for all $f \in S^{1}(G),\left\|f * \delta_{x}\right\|_{S}=\|f\|_{S}(x \in G)$, and the map $x \mapsto f * \delta_{x}, G \rightarrow S^{1}(G)$, is continuous.

Note that every symmetric Segal algebra on $G$ is a symmetric abstract Segal algebra in $L^{1}(G)$; see [16, p. 19, Proposition 1]. We refer to [16, Sect. 5] for various examples of symmetric and nonsymmetric Segal algebras. Interestingly, Theorem 3.3 provides a characterization for $\phi$-biflatness of symmetric Segal algebras.

TheOREm 3.4. Suppose that $S^{1}(G)$ is a symmetric Segal algebra on a locally compact group $G$. For each $\phi_{\rho} \in \Delta\left(L^{1}(G)\right)$, the following are equivalent:

(i) $S^{1}(G)$ is $\phi_{\rho}$-biflat.

(ii) $\left(S^{1}(G), \phi_{\rho}\right)$ satisfies condition $(W)$.

(iii) $\left(L^{1}(G), \phi_{\rho}\right)$ satisfies condition $(W)$.

(iv) $G$ is an amenable group.

Proof. The implications (i) $\Rightarrow$ (ii), (ii) $\Rightarrow$ (iii) and (iii) $\Rightarrow$ (iv) follow from Theorem 2.4, Theorem 3.3 and Corollary 3.1, respectively. For (iv) $\Rightarrow(\mathrm{i})$, we note that if $G$ is amenable, then it follows from [1, Corollary 3.4] that $S^{1}(G)$ is $\phi_{\rho}$-amenable. Hence, by [17, Proposition 2.2] we conclude that $S^{1}(G)$ is $\phi_{\rho^{-}}$Johnson amenable and so is $\phi_{\rho^{-} \text {-biflat. }}$

Now, we will discuss on $\phi$-biflatness of some specific types of Segal algebras in the group algebra $L^{1}(G)$.

Let $A(G)$ denote the Fourier algebra of $G$ as defined by Eymard in [3]. Put $\mathcal{L} A(G)=L^{1}(G) \cap A(G)$ and for $f \in \mathcal{L} A(G)$ define

$$
\||f|\|=\|f\|_{1}+\|f\|_{A(G)} .
$$


Then $\mathcal{L} A(G)$ with the above norm is a Banach space. Ghahramani and Lau in [6] showed that $\mathcal{L} A(G)$ is a Banach algebra under convolution product and it has a bounded left approximate identity in $\|\cdot\|_{1}$. This is a Segal algebra in $L^{1}(G)$, called the Lebesgue-Fourier algebra of $G$. We note that if $G$ is unimodular, then $\mathcal{L} A(G)$ is a symmetric abstract Segal algebra in $L^{1}(G)$. Moreover, it is worthwhile to mention that the Weiner's algebra $M_{1}$, see [13] and the Feichtinger's Segal algebra $S_{0}(G)$, see [4] and [19] are important examples of symmetric Segal algebras. As a consequence, we have the following.

COROLlary 3.5. Let $G$ be a unimodular locally compact group and suppose that $\mathcal{B}$ is either $\mathcal{L} A(G), M_{1}$ or $S_{0}(G)$. Then for each $\phi_{\rho} \in \Delta\left(L^{1}(G)\right)$ the following are equivalent:

(i) $\mathcal{B}$ is $\phi_{\rho}$-biflat.

(ii) $\left(\mathcal{B}, \phi_{\rho}\right)$ satisfies condition $(W)$.

(iii) $G$ is an amenable group.

We finish this section with the following remark, which provides when the generalized Fourier algebra $A_{p}(G)$ and the Lebesgue-Fourier algebra $\mathcal{L} A(G)$ with pointwise product satisfy condition $(W)$.

Remark 3.6. The Figà-Talamanca-Herz algebras $A_{p}(G)(1<p<\infty)$, introduced in [5], coincides with the Fourier algebra $A(G)$ when $p=2$. The character space $\Delta\left(A_{p}(G)\right)$ consists of all evaluation functionals $\phi_{x}$ at $x \in G$; that is, $\phi_{x}(f)=f(x)$ for all $f \in A_{p}(G)$. For every $x \in G, A_{p}(G)$ with pointwise product is $\phi_{x}$-amenable [15, Lemma 3.1], and hence $\left(A_{p}(G), \phi_{x}\right)$ satisfies condition $(W)$. Moreover, Ghahramani and Lau in [6] showed that $\mathcal{L} A(G)$ with pointwise product is a Banach algebra and also is a dense ideal of $A(G)$. Therefore by Theorem $3.3,\left(\mathcal{L} A(G), \phi_{x}\right)$ satisfies condition $(W)$, for every $x \in G$.

\section{ACKNOWLEDGEMENTS}

The authors would like to thank the referee for his/her careful reading of the paper and many valuable suggestions.

\section{REFERENCES}

[1] M. Alaghmandan, R. Nasr-Isfahani and M. Nemati, Character amenability and contractibility of abstract Segal algebras, Bull. Aust. Math. Soc. 82 (2010), 274-281.

[2] M. Essmaili and M. Filali, $\phi$-amenability and character amenability of some classes of Banach algebras, Houston J. Math. 39 (2013), 515-529.

[3] P. Eymard, Làlgèbre de Fourier d́un groupe localement compact, Bull. Soc. Math. France 92 (1964), 181-236.

[4] H. G. Feichtinger, A characterization of minimal homogeneous Banach spaces, Proc. Amer. Math. Soc. 81, (1981), 55-61.

[5] A. Figà-Talamanca, Translation invariant operators in $L^{p}$, Duke Math. J. 32 (1965), 459-501. 
[6] F. Ghahramani and A. T. Lau, Weak amenability of certain classes of Banach algebra without bounded approximate identity, Math. Proc. Cambridge Philos. Soc 133 (2002), 357-371.

[7] E. Hewitt and K. A. Ross, Abstract harmonic analysis I, Springer-Verlang, BerlinNew York, 1979.

[8] Z. Hu, M. S. Monfared and T. Traynor, On character amenable Banach algebras, Studia Math. 193 (2009), 53-78.

[9] B. E. Johnson, Non-amenability of the Fourier algebra of a compact group, J. London. Math. Soc (2) 50 (1994), 361-374.

[10] E. Kaniuth, A. T. Lau and J. S. Pym, On $\varphi$-amenability of Banach algebras, Math. Proc. Cambridge philos. Soc. 144 (2008), 85-96.

[11] E. Kaniuth, A. T. Lau and J. S. Pym, On character amenability of Banach algebras, J. Math. Anal. Appl. 344 (2008), 942-955.

[12] A. T. Lau and J. S. Pym, Concerning the second dual of the group algebra of a locally compact group, J. London Math. Soc. 41 (1990), 445-460.

[13] T. S. Liu, A. van Rooij and J. K. Wang, On some group algebra modules related to Wiener's algebra $M_{1}$, Pacific. J. Math. 55, (1974), 507-520.

[14] M. S. Monfared, Character amenability of Banach algebras, Math. Proc. Cambridge Phil. Soc. 144 (2008), 697-706.

[15] M. S. Monfared, Extensions and isomorphisms for the generalized Fourier algebras of a locally compact group, J. Funct. Anal. 198 (2003), 413-444.

[16] H. Reiter, $L^{1}$-algebras and Segal algebras, Lecture notes in mathematics, 231, Springer-Verlag, Berlin, 1971.

[17] A. Sahami and A. Pourabbas, On $\phi$-biflat and $\phi$-biprojective Banach algebras, Bull. Belg. Math. Soc. Simon Stevin, 20 (2013), 789-801.

[18] E. Samei, N. Spronk and R. Stokke, Biflatness and pseudo-amenability of Segal algebras, Canad. J. Math. 62, (2010), 845-869.

[19] N. Spronk, Operator space structure on Feichtinger's Segal algebras, J. Funct. Anal. 248, (2007), 152-174.

M. Essmaili

Department of Mathematics, Faculty of Mathematical and Computer Sciences Kharazmi University, 50 Taleghani Avenue, 15618 Tehran, Iran

School of Mathematics, Institute for Research in Fundamental Sciences (IPM) P.O.Box 19395-5746, Tehran, Iran

E-mail: m.essmaili@khu.ac.ir

M. Rostami

Faculty of Mathematical and Computer Science

Amirkabir University of Technology

424 Hafez Avenue, 15914 Tehran

Iran

E-mail: mross@aut.ac.ir

M. Amini

Department of Mathematics, Faculty of Mathematical Sciences

Tarbiat Modares University, 14115-134 Tehran, Iran

School of Mathematics, Institute for Research in Fundamental Sciences (IPM)

P.O.Box 19395-5746, Tehran, Iran

E-mail: mamini@modares.ac.ir

Received: 17.11.2014.

Revised: 28.6 .2015$. 